\title{
Solutions for Planning Smart Hybrid Public Transportation System - Poznan Agglomeration as a Case Study of Satellite Towns' Connections
}

\author{
Marzena Banach ${ }^{1}$ Katarzyna Kubiak $^{2}$ and Rafał Długosz ${ }^{3}$ \\ ${ }^{1}$ Poznan University of Technology, Institute of Architecture and Spatial Planning, \\ ul. Nieszawska 13C, 61-021 Poznan, Poland \\ E-mail: marzena.banach@erba.com \\ 2 Adam Mickiewicz University \\ Faculty of Mathematics and Computer Science \\ ul. Umultowska 87, 61-614 Poznan, Poland \\ E-mail: kbkubiak45@gmail.com \\ ${ }^{3}$ UTP University of Science and Technology \\ Faculty of Telecommunication, Computer Science and Electrical Engineering \\ ul. Kaliskiego 7, 85-796, Bydgoszcz, Poland \\ E-mail: rafal.dlugosz@aptiv.com, rafal.dlugosz@gmail.com
}

\begin{abstract}
A primary goal of the Internet of Things is to create smart spaces, including smart cities and intelligent transportation system (ITS). One of the ITS variants is smart hybrid public transportation system, whose efficient development requires a broad support from the side of information and communication technologies (ICT). For such a system to function optimally, vehicles that are part of it have to be able to exchange data with other road users and road infrastructure. This applies especially to autonomous vehicles, whose trajectories may be in the future controlled through the vehicle-to-infrastructure (V2I) communication network. In the paper we propose a system that can be used to optimize the boundaries of relatively small suburban zones, in which public autonomous vehicles would be allowed to operate. Vehicles of this type may offer the transport only to the nearest local railway stations, from which the travel to the central city of the agglomeration would be continued for example by train. The concept of the proposed solution is based on the Voronoi diagrams, in which particular suburban train stations are treated as local attractors. The proposed software system in one of its stages uses Google Maps engine that allows for the determination of road distances and travel times between particular towns. On the basis of such data it determines the mentioned zones. Their boundaries, as well as optimal routes in a given period of the day may be communicated to the vehicles through the V2I system. The system performance is presented for an exemplary case of Poznan city, Poland.
\end{abstract}

Keywords-Intelligent Public Hybrid Transport System, Transport Planning, Autonomous Vehicles, Software System

\section{INTRODUCTION}

The idea of smart cities is frequently connected with technical and social solutions which aim at improving the quality of broadly understood everyday functioning of city dwellers. These solutions include various facilities, in which novel artificial intelligence (AI) as well as information and communication technologies (ICT) are used as a basis [1]. For example, these technologies are used to facilitate the access to information, offering information systems for passengers, tourists, etc. On a higher level, more advanced solutions are proposed such as remote access to offices [2]. However, one of the main pillars of smart cities is the so-called intelligent transportation system (ITS) [3], which bases on various AI and ICT technologies to a high extent. The ITS is expected to transform the transport toward more functional, flexible and ecological solutions. A truly intelligent system of this type should offer comprehensive solutions at the level of larger areas such as city agglomerations. This aim can be accomplished by developing an intelligent public hybrid transportation system.

Technological solutions used in smart cities should primarily lead to real social benefits in the areas of safety, ecology and economy. With appropriate approach, these benefits can be directly offered by the ITS. One of them, which is the topic of this work, is to improve the competitiveness and flexibility of public transport in towns and villages - satellites of major cities in agglomerations. Their inhabitants, who constitute a significant percentage of all inhabitants of the agglomeration, regularly commute to the central city, mostly for educational and occupational purposes. The lack of an attractive public transport offer, e.g. rare and inconvenient connections, long travel time, causes the car to be a frequently used means of transport (in Poland). It has lead to a growing concern in recent years, which is increasing city traffic which adversely affects the quality of life.

Not unlike most areas of life, transport is subject to market competition. In terms of public transport, a competitive offer is such an offer which is comparable to what individual transport provides. Simply speaking, people demand faster, cheaper, more convenient and more flexible solutions.

One of the solutions to the described problem is to use a combination of two means of transport: own car and public transit. This idea is not new. It has been promoted for many 


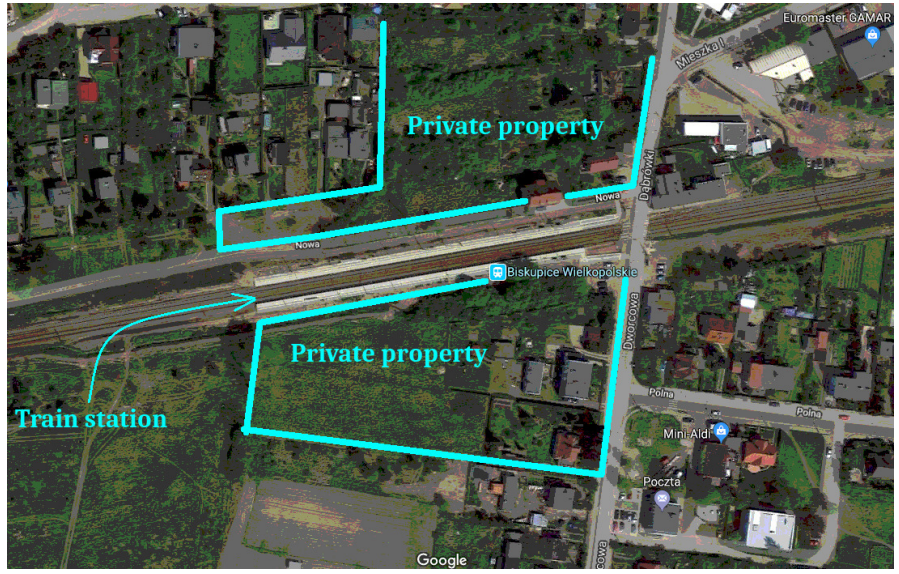

Fig. 1. The environment of the Biskupice Wielkopolskie train station $-20 \mathrm{~km}$ from Poznan city, Poland.

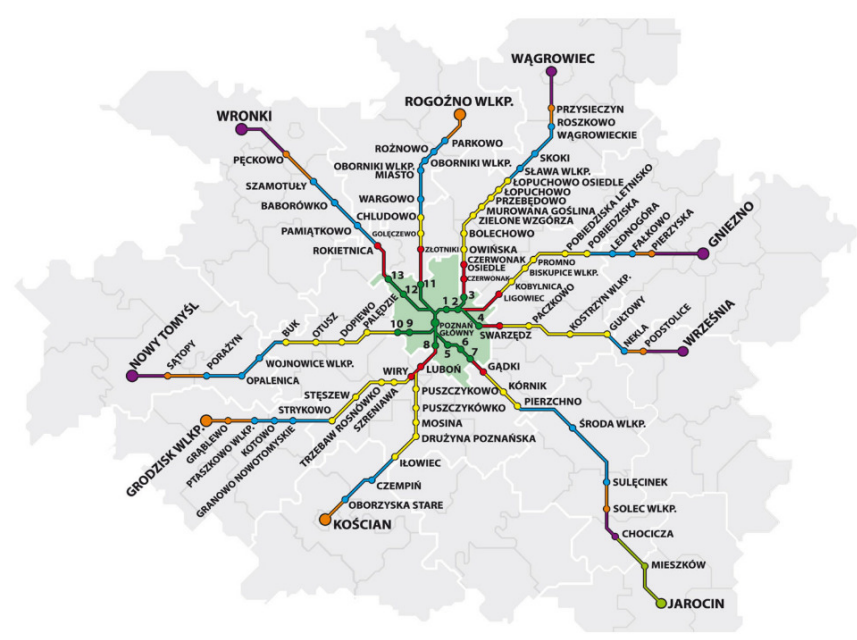

Fig. 2. Public hybrid transport system in Poznan [4].

years in numerous agglomerations around the world. Usually, parking lots are created close to a train station and are available to people who change to a train. However, it is not always possible in practice. For example, there is no room for a sufficiently large parking lot at many railway stations in Poland. Figure 1 presents the railway station in Biskupice Wielkopolskie, a village located about $20 \mathrm{~km}$ from Poznan (Poznan is the central city of the agglomeration). It is not possible to build a parking lot at this station due to the lack of space. Biskupice Wielkopolskie and neighboring villages are inhabited by more than 2,000 people, many of whom travel to Poznan almost every day. This means even more than hundred additional cars on the roads of Poznan. It is a relatively common problem in Poland which has to be solved.

In recent years, the automotive industry has been dynamically developing. One of the hot directions is to popularize autonomous vehicles, developed by many car manufacturers. The aim of such solutions is primarily to improve road safety through the elimination of mistakes made by drivers. Another important goal is to optimize the driving style, which will lead to the reduction in pollutant emissions.
However, it is worth to pay attention to a few basic aspects related to autonomous vehicles. One of them is the large complexity of the environment "seen" by such vehicles. In cities, for example, it means a large number of objects (pedestrians, other vehicles, bicycles) in the vicinity of the car which have to be tracked by its systems. Another problem can be a long transition period, during which autonomous vehicles will coexist with conventional cars, which raises safety issues. In spite of these problems, the emergence of autonomous vehicles may have positive impact on public hybrid transport. With some assumptions, such cars can be used in the described transition period. We consider using such cars in limited, well defined and flexible as well as less dense suburban areas.

In recent years, much attention has been paid to public hybrid transport, introduced in many cities. The aim is to achieve such flexibility of this transport that possibly many residents will be encouraged to give up using own cars. A joint tariff and joint schedule plans take into account buses, trams, subway (if available), urban and suburban trains. In urban literature, the problem of the so-called first and last kilometer is highlighted. According to this rule, if reaching the nearest public transport stop consumes too much time (over 10 minutes $/ 1 \mathrm{~km}$ ), the residents usually choose their own car. New concepts of compact cities with buildings located near to public transport stops will satisfy most of the needs of the inhabitants, so that it would not be necessary to cover long distances frequently. One of the basic goals of this concept is to eliminate the need to use own car on a daily basis.

The solutions discussed in this work are not aimed at polemics with the idea of compact cities postulated by modern urbanism. The main goal is to facilitate the access to the city resources, including the case of wider agglomerations. Optimization of public transportation using autonomous vehicles (taxis) outside central cities can shorten the access time to the city resources, while maintaining ecological goals. One can talk here about an apparent shortening of the distances.

As mentioned above, the idea of the hybrid public transportation system is known and developed for many years over the world. New trends in this area, however, are various concepts of the use of autonomous vehicles in public transport. One of the solutions here are autonomous taxis already used in some cities (e.g. NuTonomy in Singapore). In the scope of our interests, on the other hand, are technical solutions that aim at optimizing public transport away from the major cities of the agglomerations [5]. We think this will have a positive impact on the overall area of particular agglomerations. Such optimization can be understood, for example, as minimizing the total distances traveled by the inhabitants of particular suburban areas by private cars. At the same time, the goal is to keep the flexibility offered currently by own means of transport in these areas to the highest possible level $\rightarrow$ minimizing the total travel time. In this work, we deal with technical solutions for a system that can be used for planning the hybrid transport in suburban area. In particular, we focus on defining boundaries of particular zones available for selected groups of autonomous vehicles. To illustrate the complexity of the problem, it is worth mentioning that there are over 100,000 cities and towns in Poland that need to be included in planning. 

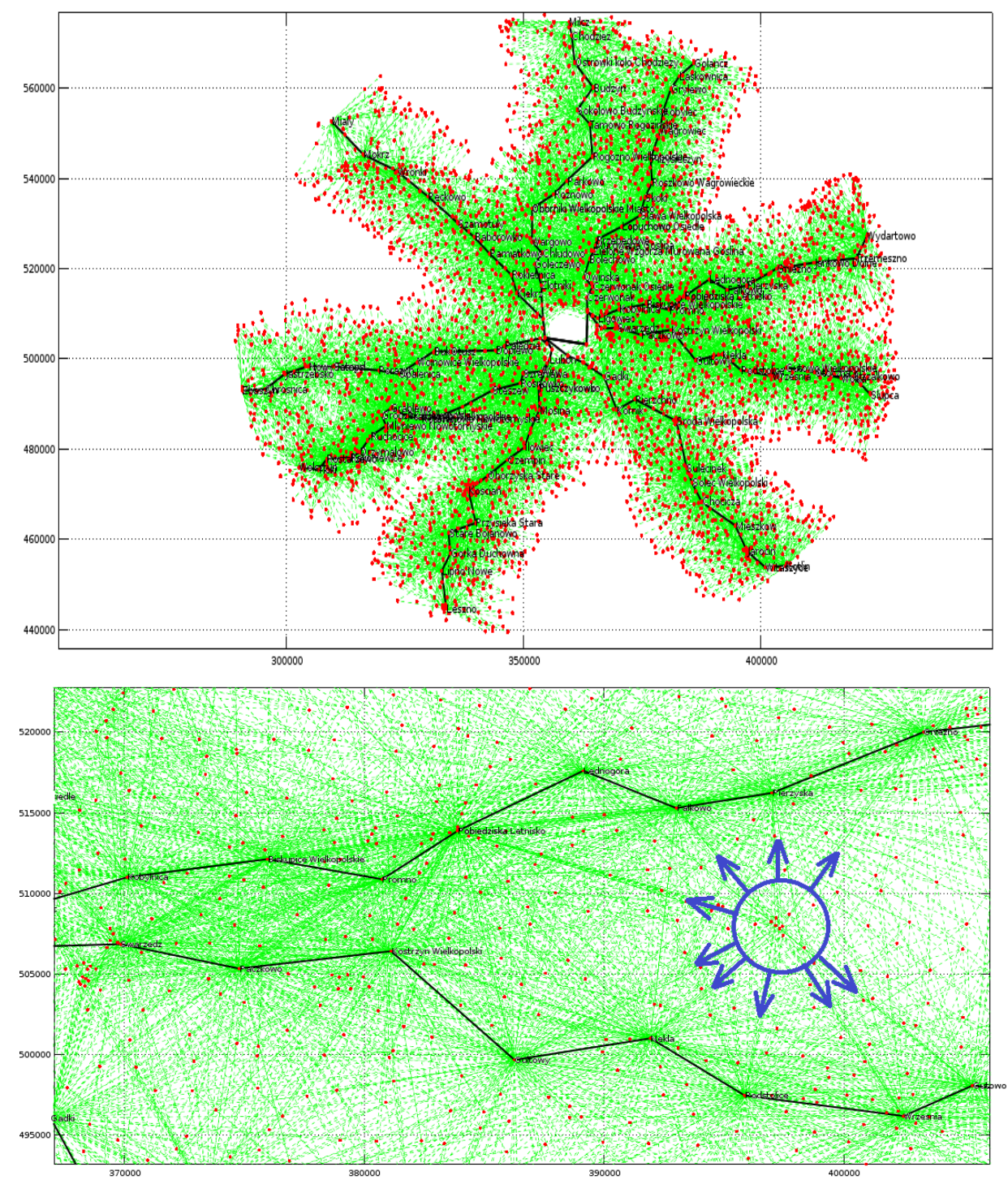

Fig. 3. Illustration of data stored in the DT2 $2_{l, s}$ datasets: selected settlement-to-station assignments (up). Exemplary area belonging to ROIs of nine stations for $r_{\max }$ set to $22 \mathrm{~km}$ (bottom).

The paper consists of several parts. The next section presents a short literature study related to the subject of the work. We briefly discuss public hybrid transport systems and the use of Voronoi diagrams for agglomerations. In the following section we describe the proposed software system, as well as exemplary results for the Poznan agglomeration in Poland. The conclusions are formulated in last section.

\section{StATE-OF-THE ART STUDY AND RESEARCH METHODOLOGY}

The investigation results presented in this work are for an exemplary case of the Poznan agglomeration. However, the proposed software system can be quickly configured and used for any agglomeration. The methodology applied in our investigations partially results from the following state-of-the art study which showed us the areas of the application of the system.

\section{A. Public hybrid transport systems in Poznan}

Combining different means of transport is an increasingly common practice, especially in larger urban agglomerations. The common tariff plan connects buses, trams, subway and suburban trains wherever possible. This combination also includes the synchronization of timetables so as to improve the smoothness of getting around the city. An example of these solutions is the transport system in the Poznan agglomeration. In this case, the BTK (bus, tram, train) offer extends within a radius of approximately $50 \mathrm{~km}$ from Poznan, as shown in Fig. 2. The investigation results, presented in next section, were conducted for a similar area.

\section{B. Using Voronoi diagrams}

The proposed work is based on the method of dividing the space into 'zones of influence', which are often illustrated with the help of the Voronoi diagrams. This method is also 


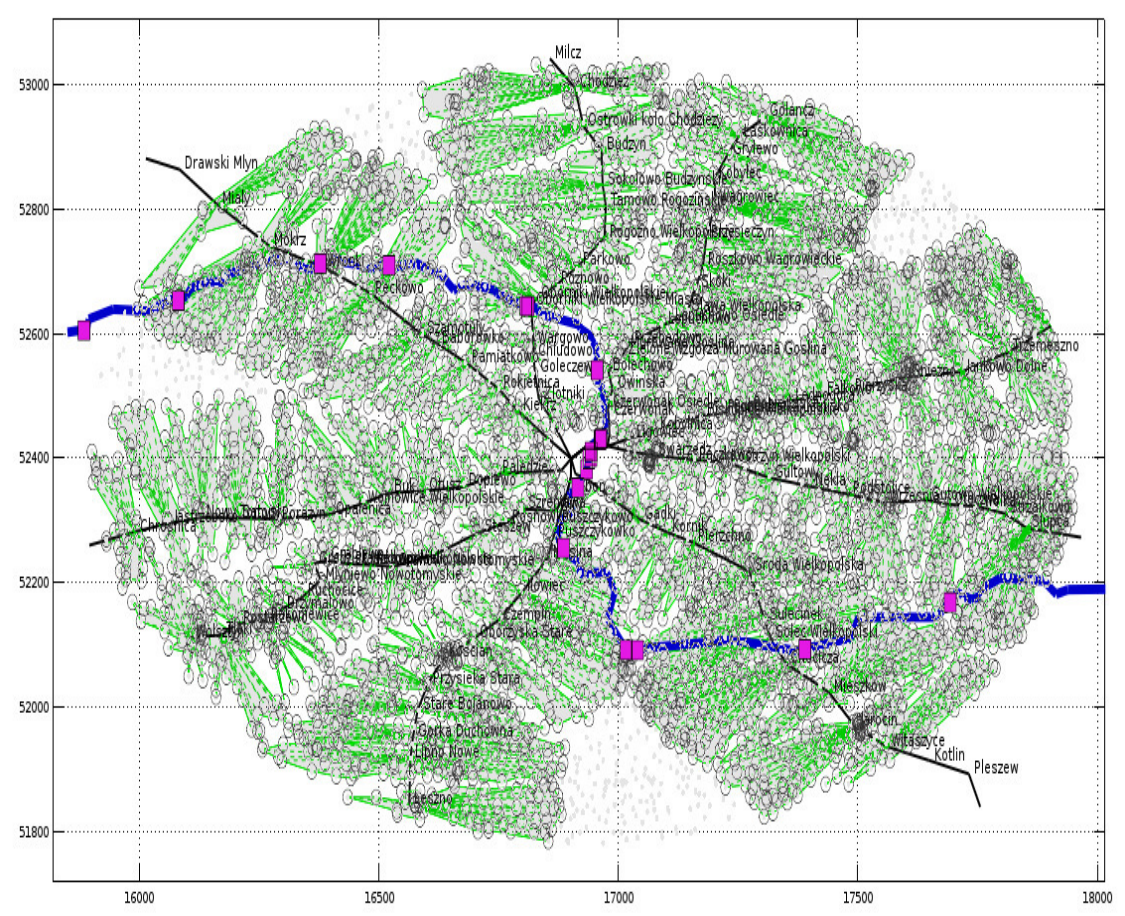

Fig. 4. An exemplary Voronoi diagram created by the proposed system of the overall Poznan agglomeration for the radius of $50 \mathrm{~km}$.

used in urban planning [6] - [10]. An interesting example of using this methodology for planning the public transport is reported in [11]. In [11] a station location method for a commuter rail system has been demonstrated for an example case of Brasilia Metropolitan Area. This case is somehow similar to what we observe in Poland - traveling even large distances due to, for example, job opportunities in larger cities. The authors of [11] in their work focus on planning new rail routes. We investigate a different problem. We treat existing railway stations as reference points, while we are interested in optimizing access to particular stations from nearby towns.

\section{Input data for the investigation}

To make the investigations comprehensive and to increase the reliability of the tests of the implemented system, we used an initial dataset consisting of parameters of 120,000 Polish cities, towns and villages. Every entry provides the name of the unit, its county and its geographical location (longitude and latitude), among others. It was used to determine which settlements lie within a given radius from Poznan and will be denoted as DT0 from now on.

\section{PROPOSED SYSTEM AND INVESTIGATION RESULTS}

We used the following notation to denote the datasets used in calculations: capital letters indicate the type of data stored, while the following number indicates the step of calculations. Also, each step has input and output data, as shown in Table I.

\section{Step 1: Selection of units belonging to a given agglomera- tion}

The aim of the first step is to identify the units from the input dataset DT0 whose straight line distance (calculated from
TABLE I

INPUTS AND OUTPUTS OF PARTICULAR COMPUTATION STEPS (EXPLANATION OF ABBREVIATIONS IN TEXT).

\begin{tabular}{|c|c|c|}
\hline Step No. & Inputs & Outputs \\
\hline 1 & DT0 & DT1 \\
\hline \multirow{2}{*}{2} & DT1 & DT2 $2_{l, s}$ \\
\hline 3 & $r_{\min }, r_{\max }$ & DT $3_{l, s}$ \\
\hline 4 & $\mathrm{DT} 2_{l, s}$ & DV4 \\
\hline 5 & $\begin{array}{c}\mathrm{DT} 3_{l, s} \\
\text { DT1 }\end{array}$ & Voronoi diagrams \\
\hline
\end{tabular}

their geographical coordinates) from Poznan is less or equal to $50 \mathrm{~km}$. This value is based on the reach of the abovementioned BTK transport system. This step was implemented in the $\mathrm{C}++$ language. The resultant dataset is composed only of nearly 4,000 settlements located in the investigated area. It will be denoted as DT1 from now on.

\section{Step 2: Building regions of interest for particular train stations}

Firstly, we created a dataset, denoted as DR1, of subsequent railway stations in the investigated area and their geographical coordinates. They are grouped into 9 train lines leading to Poznan. In order to simplify the following computations, every line and every station within a line have their own identification numbers, denoted ad ID. Consequently, the DR1 dataset provides the so-called local central points/local attractors which gather road traffic from the nearest settlements. 
(a)
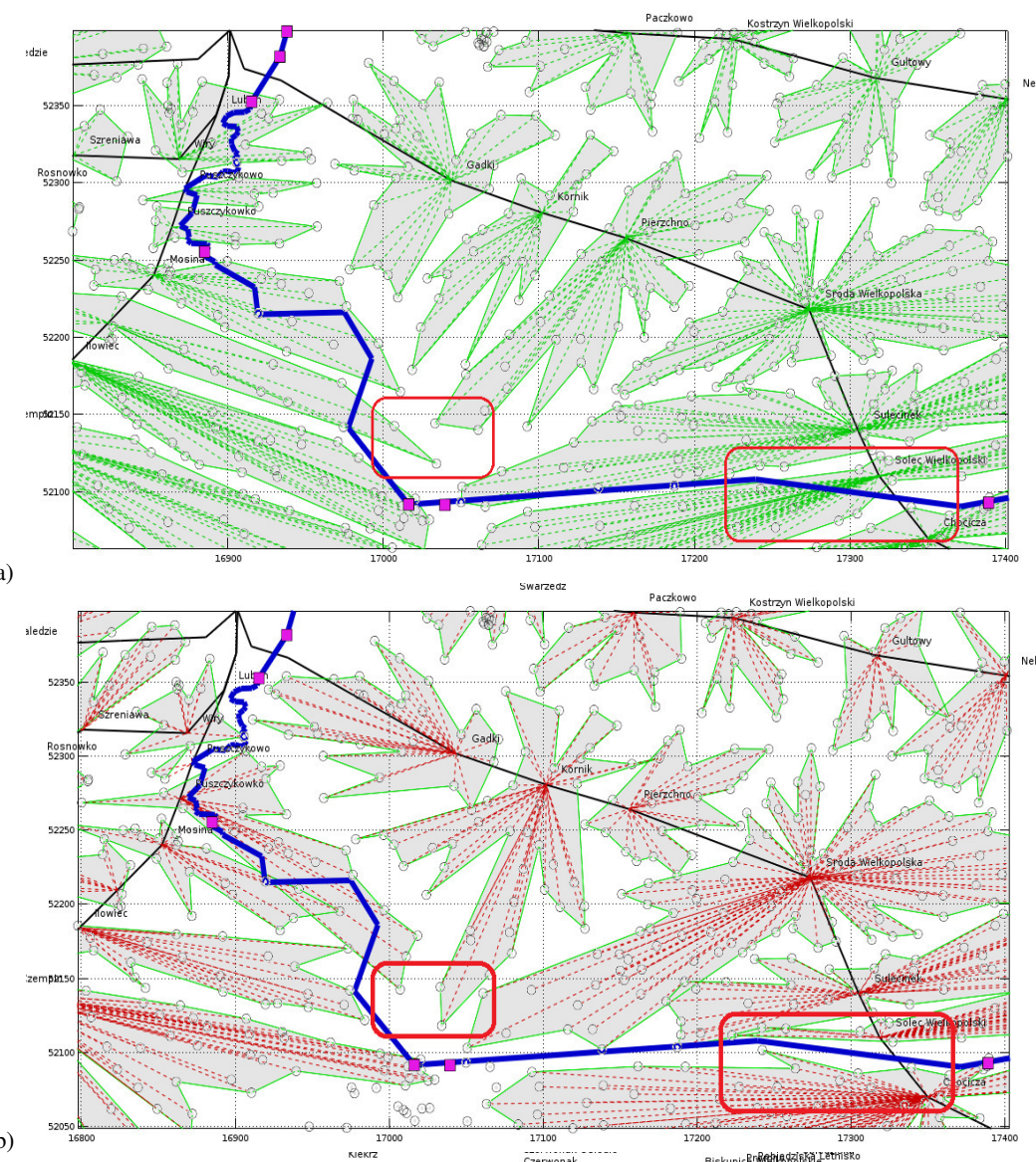

(b)

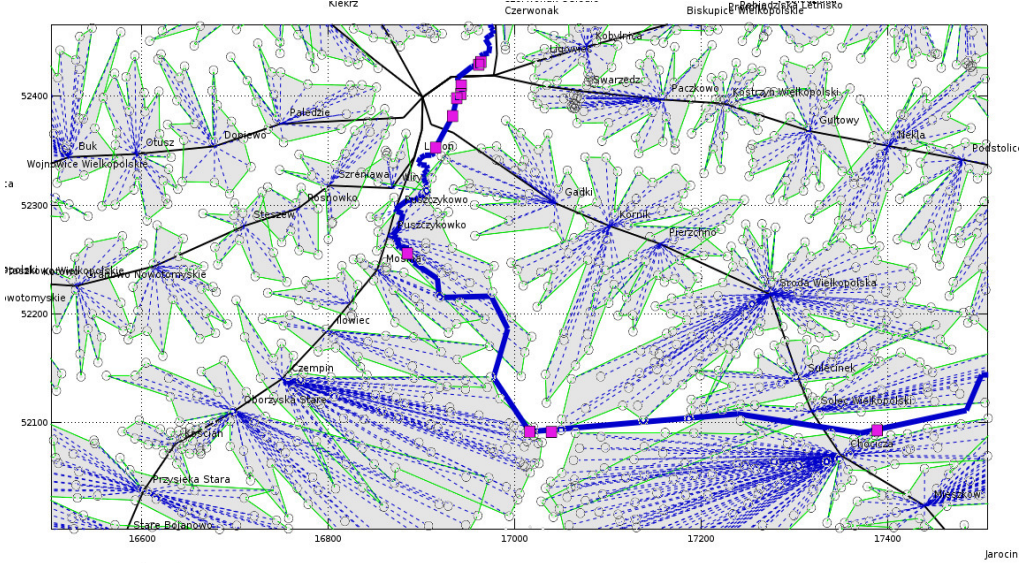

Fig. 5. Voronoi diagrams of selected areas for geographical (a), road (b), and travel time distances (c). The wide blue line is the Warta river, while the magenta blocks are locations of bridges

Then, we define a circular region of interest, denoted as ROI, for every railway station from DR1. ROI is described by two parameters the minimum radius $r_{\min }, r_{\min }>0$ and the maximum radius $r_{\max }$. Selecting a non-zero value of the $r_{\min }$ is necessary to exclude internal districts of cities, in which particular train stations are located, from further computations.

Finally, for each station from DT1 a dataset DT $2_{l, s}$ is created, where $l$ is the ID of a given train line, and $s$ is the ID of a given station. Every resultant set DT2 $2_{l, s}$ is composed of villages located in the ROI of station $s$ from line $l$ and straight line distances from the villages to the station. It is worth to notice that particular ROIs may overlap to some extent.
The values of $r_{\min }$ and $r_{\max }$ were selected to be $3 \mathrm{~km}$ and $22 \mathrm{~km}$, respectively. Larger values of $r_{\max }$ did not lead to significant changes in the results, i.e. shapes of particular areas in the obtained Voronoi diagrams. However, larger values of $r_{\max }$ significantly increase the number of operations in the subsequent steps. The larger the value of $r_{\max }$ is, the higher the extent to which particular ROIs overlap. Consequently, a given settlement belongs to a bigger number of ROIs. Selected results are shown in Figure 3. Dashed lines connect particular towns with railway stations. This step was also conducted in $\mathrm{C}++$. 


\section{Step 3: Computation of distances according to road map}

In the third stage (JavaScript), the system added two additional pieces of information to DT $2_{l, s}$ datasets, i.e. the distance along the road and the travel time from each settlement to its station, thus creating respective $\mathrm{DT} 3_{l, s}$ datasets. It was achieved with the use of Google getDistanceMatrix() service. More than 40,000 combinations were checked for different values of the parameter $r$. The part of the system developed in JavaScript strongly facilitated the procedure. As the getDistanceMatrix() allows to check only 25 combinations in a single run, the system split the DT $2_{l, s}$ datasets into smaller subsets and provided them to the Google service in the appropriate way (timing).

The resultant $\mathrm{DT} 3_{l, s}$ datasets provide three values for each settlement-to-station assignment: (i) straight line distance based on geographical coordinates (GEO), (ii) distance measured along the road network (GGLD - GooGLe Distance), (iii) travel time (GGLT - GooGLe Travel).

\section{Step 4: Obtaining the final dataset}

Now, for each settlement the system determines the nearest station in regard to three distance criteria: GEO, GGLD and GGLT, combining information from DT1 and DT $3_{l, s}$ to form the final DV4 datasets for each settlement. This computation process may be regarded as filtering with the Min() function.

\section{Step 5: Building Voronoi diagrams}

In this step (Octave environment), Voronoi diagrams are finally built for the three above-mentioned criteria. The resultant diagram of the whole Poznan agglomeration for the radius of $50 \mathrm{~km}$ is shown in Fig. 4. Fig.5 shows selected areas covered by Voronoi diagrams in regard to the GEO (a), GGLD (b) and GGLT (c) criteria. The results are presented in a coordinate system in which latitude and longitude are coordinate axes in order to provide a legible diagram. Considering a relatively small area (in global sense) of the Poznan agglomeration, it does not introduce noticeable distortions.

\section{DiscusSiOn OF RESULTS}

As expected, Voronoi diagram for the GEO criterion unambiguously assigned areas which do not overlap. For the two remaining criteria, particular areas may overlap while being visualized according to geographical coordinates. It is a natural effect, as the roads increase the distances, causing local nonlinearities.

Also, Figs. 4 and 5 show the Warta river (thick blue line) which is a natural barrier affecting the shape of Voronoi diagrams. Bridges enabling road traffic are also marked. Exemplary areas on which the river presence results in the assignment of some settlements to different ROIs.

On the basis of such Voronoi maps, it is possible to estimate the boundaries of areas in which autonomous vehicles could be used in public hybrid transport in the future. Factors that should be taken into account are not only the distances to particular railway stations, but also the travel time. An important factor is the total travel time which also includes the train journey to the central city. It is possible that the nearest railway station is located further from the central city, which will additionally increase the total travel time.

One of the possibilities is introducing dynamic shaping zones served by autonomous vehicles based on the travel time, which can be varied during the day, as well as the communication schedule for particular railway lines.

\section{CONCLUSIONS}

The paper presents an idea of improving public hybrid transport system in the future based on autonomous vehicles. Such cars could be appropriate in areas with less traffic, outside of the central city. The goal is to decrease the number of vehicles in already crowded cities by making public transport more flexible.

Another important issue is the appropriate division of the agglomeration area into zones so as to minimize the total distances traveled by autonomous vehicles in a given area. For this purpose, we designed a software system which determines these zones in regard to both road distance and travel time, basing on publicly available data.

The presented results, considered by us as preliminary, are a stage of a larger project. The purpose of the project is to facilitate the planning of public transport in general.

\section{REFERENCES}

[1] I. Azkuna, "Smart cities studies: International study on the situation of ICT, innovation and Knowledge in cities," The Committee of Digital and Knowledge-based Cities of UCLG, Bilbao, 2012.

[2] Spot Mairie, [Online]. Available: http://www.nicecotedazur.org/ la-metropole/spot-mairie, (access 2019.05.15)

[3] C. Benevolo, R. P. Dameri, B. D’Auria, (Editors: T. Torre, A.M. Braccini, R. Spinelli), "Smart Mobility in Smart City. Action taxonomy, ICT intensity and public benefits," Empowering Organizations: Enabling Platforms and Artefacts, Springer, ISBN 978-3-319-23784-8, 2016, pp. 13-28.

[4] Bus - Tram - Railway System in Poznan. [Online]. Available: http://bustramwajkolej.pl/

[5] M. Banach, "Use of Intelligent hybrid solutions in sustainable public transportation system for the net of small cities", Zarzqdzanie Publiczne (Public Management), No. 3 / 2019

[6] A. Nowak, "Application of Voronoi diagrams in contemporary architecture and town planning" Challenges of Modern Technology, Vol. 6, no. 2, pp.30-34, 2015.

[7] M. Abellanas, B. Palop, "Urban Data Visualization with Voronoi Diagrams," In: Gervasi O., Murgante B., Laganà A., Taniar D., Mun Y., Gavrilova M.L. (eds) Computational Science and Its Applications ICCSA 2008. ICCSA 2008. Lecture Notes in Computer Science, vol 5072. Springer, Berlin, Heidelberg

[8] F. Bahraminejad, K. Babaki, "Application of Voronoi diagram as an architectural and urban planning design tool," Indian Journal of Fundamental and Applied Life Sciences, ISSN: 22316345, 2015 Vol.5 (S1), pp. 1776-1783, [Online]. Available: www.cibtech.org/sp.ed/jls/2015/01/jls.htm

[9] J. Park, S. Ji, H. Jun, "The Application of Voronoi Diagram into the Space Planning for Urban Design," 7th International Symposium on Architectural Interchanges in Asia (ISAIA 2008), pp. 524-528.

[10] Z. Xinqi, W. Shuqing, F. Meichen, Z. Lu, Y. Shujia, (2010) "Urban Cluster Layout Based on Voronoi Diagram," In: Elleithy K. (eds) Advanced Techniques in Computing Sciences and Software Engineering. Springer, Dordrecht

[11] D. R. Mota, M. Takano, Pastor W. Gonzales Taco, "A Method Using GIS Integrated Voronoi Diagrams for Commuter Rail Station Identification: A Case Study from Brasilia (Brazil)," Procedia - Social and Behavioral Sciences, Volume 162, 19 December 2014, pp. 477-486. 